\title{
Journal of General Medicine and Clinical Practices
}

David Mulenga ${ }^{1 *}$. J General Medicine and Clinical Practices http://dx.doi.org/ 10.31579/1.10010

\section{Impact of Cooking Fuel Choices on Maternal Lung Functions in Selected} Rural and Urban Areas of Copperbelt Province, Zambia

David Mulenga $^{1^{*}}$, Seter Siziya ${ }^{1}$.

${ }^{1}$ Department of Clinical Sciences, Copperbelt University, Michael Chilufya Sata School of Medicine, Public Health Unit, Ndola.

${ }^{*}$ Corresponding Author: David Mulenga, Department of Clinical Sciences, Copperbelt University, Michael Chilufya Sata School of Medicine, Public Health Unit, Ndola. E-mail: davykdn@gmail.com

Received date: June 06, 2018; Accepted date : June 21, 2018; Published date: July 02, 2018.

Citation this Article : David Mulenga, Seter Siziya, Impact of Cooking Fuel Choices on Maternal Lung Functions in Selected Rural and Urban Areas of Copperbelt Province, Zambia, J General Medicine and Clinical Practices. Doi: http://dx.doi.org/ 10.31579/1.10010.

Copyright : (c) 2018.David Mulenga. This is an open-access article distributed under the terms of the Creative Commons Attribution License, which permits unrestricted use, distribution, and reproduction in any medium, provided the original author and source are credited.

\begin{abstract}
Background

Considering the respiratory health risk of exposure to biomass cooking fuel emissions, this study was conducted to elucidate the relationship between cooking fuel choices and declined pulmonary function in rural and urban population in the Copperbelt Province of Zambia.
\end{abstract}

\section{Methods}

We carried out a cross-sectional study of 1,170 healthy nonsmoking pregnant women from Masaiti and Ndola predominantly using biomass fuel for cooking. Questionnaire based data was acquired along with standardized measures of lung function. MIR Spirobank G (Italy) was used in spirometry based on American Thoracic Standards.

\section{Results}

The present study found that over two thirds $(69.2 \%)$ of pregnant women in the study population use biomass for cooking and only $12.4 \%$ use electricity only. Declined lung function was found to be statistically significantly associated with cooking fuel choices ( $\mathrm{p}$ - value 0.005$)$ and a weak association was observed with gravida at a p-value of 0.056 . Pregnant women using crop residues as cooking fuel were two times more likely to have a declined lung function [AOR $2.33(1.27,4.30)]$ compared with pregnant women using mixed fuel type (biomass and electricity) and those using electricity only were $57 \%$ less likely to have a declined lung function [AOD $0.43(0.26,0.69)]$.

\section{Conclusion}

Use of biomass for cooking among pregnant women is a strong determinant of declined lung function. Interventions to improve maternal respiratory health outcomes in Zambia and other countries in subSaharan Africa should involve making cleaner cooking fuel energy options available and accessible by ordinary women from both rural and urban areas.

Keywords

Cooking fuel, Lung function, Biomass .

\section{Introduction}

In developing countries, use of fuels such as wood, dung and crop residue is estimated in as many as $70 \%$ of households $[1,2]$ or in more than 3 billion people worldwide according to other researchers[3]. According to Chidumayo et al and Indaba Agricultural Policy Research Institute (IAPRI), charcoal is widely used in urban Zambia as a source of cooking fuel, either on its own or in combination with electricity, it's further been observed that even among electrified households, charcoal is commonly used in combination with electricity, more than electricity only, an indication of pervasive fuel stacking, rather than fuel switching [4,5]. Health problems related to the use of solid fuels as an energy source have been an issue of concern especially in many poor countries. Biomass fuels are at the low end of the energy ladder in terms of combustion efficiency and cleanliness [6]. they emit large quantities of smoke that contains many noxious components [7]. Periodic and longtime exposure to solid fuel emissions may lead to various types of adverse health outcomes such as chronic bronchitis, increase in the risk of tuberculosis, respiratory failure and cor pulmonale [8].

It is for this reason that biomass use for cooking and heating houses has been implicated in respiratory function disorders and pulmonary diseases due to emissions from incomplete combustion in usually poorly ventilated houses. The cheaper cooking fuel options in any context are generally less efficient cooking fuels, emit more smoke, and are the ones used by groups of people with the most poorly designed houses. Fuels such as propane, liquid petroleum gas (LPG), or ethanol usually burn very cleanly, but remain too expensive for many households. 
Electricity is the least polluting type of energy for domestic use provided households are geographically separated from power stations, but the problem is that it is not affordable to the average population.

According to Rehfuess, descending from the energy ladder to cheaper types of cooking fuel such as charcoal, wood and dung or crop residues, there are polluting fuels with both poor combustibility and highly toxic emissions. Simple muddy, thatched homes sometimes built with animal skins rarely have a chimney and, if present, the chimney is often a simple vent with no air-drawing flue [9]. Combustion of cheaper fuels in homes is responsible for indoor air pollution and indoor air pollution is in turn responsible for almost 2 million annual deaths and accounts for $2.7 \%$ of the global burden of disease [10]. Fuel types determine the amount of pollutants that would be emitted for instance, more pollutants are expected from fuels at the bottom of the energy ladder such as wood, cow dung and crop residue because they are unprocessed energy sources. The emissions have an adverse effect on the lung functions of the exposed individuals. Lower $\mathrm{FEV}_{1}$ and FVC values have been reported [11] among people predominantly using biomass compared with those using other fuel types. This observation is attributed to the effects of pollutants emitted by unprocessed cooking fuel types [12] on lung functions. Therefore, cooking fuel choices are an important predictor of reduced lung function among exposed populations and this has been demonstrated by many other researchers. In a study to evaluate the effects of solid fuels smoke exposure on pulmonary function, researchers observed a significant reduction in FVC, FEV $1, \mathrm{FEV}_{1}$ /FVC ratio and forced expiratory flow 25-75 in solid fuel users compared to individuals who used liquefied petroleum gas[13].

Biomass combustion causes high levels of health-damaging indoor air pollution including carbon monoxide, particulate matter, nitrogen dioxide and polycyclic aromatic hydrocarbons [14]. Since studies consistently show high indoor air pollution levels in households using biomass with PM2.5 being observed to be 10 to $>50$ times the WHO annual average Air Quality Guideline level [15], it therefore, follows that cooking fuel choices are a good proxy for noxious pollutants especially particulate matter (PM2.5). Many researchers have demonstrated that biomass combustion emits PM2.5 and many other pollutants. Some researchers in resource constrained countries do not directly measure exposure to household air pollution due to challenges in acquiring indoor air pollutant measurement devices. They use the effect estimates which are based on exposure determined by fuel type such as "use of solid fuels" or "exposure to biomass" compared with "use of other fuels" [12]. The proxy factors used to estimate level of exposure include involvement in cooking during pregnancy , use of charcoal or wood for cooking [16,17,18], and cooking in an enclosed kitchen with no window present [19].

To demonstrate the impact of solid fuel on the lung function, Desalu et al recruited women who predominantly used solid fuels for cooking in the study and findings indicated a reduction in pulmonary function [20]. It is important to note that reduction in pulmonary function due to the effect of solid fuel has not been documented consistently by all investigators. In a previous study to assess the impact of different cooking fuels on pulmonary function, Rinne et al. observed no difference in lung function among the different fuel types

[11]. Another previous study by Reddy et al. also demonstrated that cooking with biomass was not significantly associated with lung function decline. However, this unexpected result was attributed to good ventilation in the kitchen where cooking was conducted. Inefficient ventilation of the kitchen is known to concentrate pollutants leading to adverse effects of biomass smoke on lung function [21]. We therefore, conducted this study in order to assess the impact of cooking fuel choices on maternal lung functions among Masaiti and Ndola residents in Zambia.

\section{Methods and Materials}

A cross sectional study among 1170 pregnant women in Masaiti and Ndola was conducted and a structured questionnaire was used to obtain information on background characteristics. Pulmonary function tests were performed at least three times for each pregnant woman in a sitting position with closed nostrils using MIR - Spirobank G (Italy) spirometer and with a different mouthpiece for each subject. The spirometer was calibrated to suit individual participant according to sex, age, height and weight. Demonstration about the test was done for the pregnant woman before the test. The tests were performed according to American Thoracic Society (ATS) standards. Spirometry values (FEV1, FVC and FEV1/FVC) were taken three times and the best result of the three measurements was recorded.

\section{Data processing and analysis}

Spirometry parameters of interest were FVC, FEV1 and FEV1/FVC. FVC and FEV1 < than $80 \%$ was regarded as a declined or poor lung function and similarly, FEV1/FVC ratio of $<$ than $70 \%$ was also regarded as a reduced lung function. Preliminary analysis involving bivariate analysis was conducted in Epi Info and then the Multivariate Logistic Regression was carried out to determine the cooking fuel type that determines poor lung function. The magnitudes of association were estimated using odds ratios and their $95 \%$ confidence intervals. Statistical significance level was set at $5 \%$.

\section{Ethical Consideration}

The Tropical Disease Research Centre (TDRC) Ethics Committee approved the study and participants signed a consent form to participate in the study.

\section{Results}

\section{Socio-economic and demographic characteristics of the pregnant women}

Out of a total of 1,170 pregnant women recruited to the study, more than half of them (58.9\%) were from the urban area (Ndola City) and the rest $(41.1 \%)$ from the rural area (Masaiti District). More than $80 \%$ of the study population were between the age of 35 and 16 years old with the highest proportion $(35.7 \%)$ belonging to the age group 20-25 years old. Almost everyone $(99.3 \%)$ practiced Christianity. Slightly over half of the participants when put together belonged to two provinces, namely northern $(31.2 \%)$ and eastern $(20.4 \%)$ provinces of Zambia. Majority $(91.2 \%)$ of the participants were in a marriage relationship with more than half of the women (54.6\%) having attained secondary and a third (30.0\%) primary level of education. Over two thirds of the pregnant women (69.6\%) were unemployed housewives and $13.7 \%$ were traders. Table 1 gives a summary of the demographic and socio-economic characteristics of the population. 


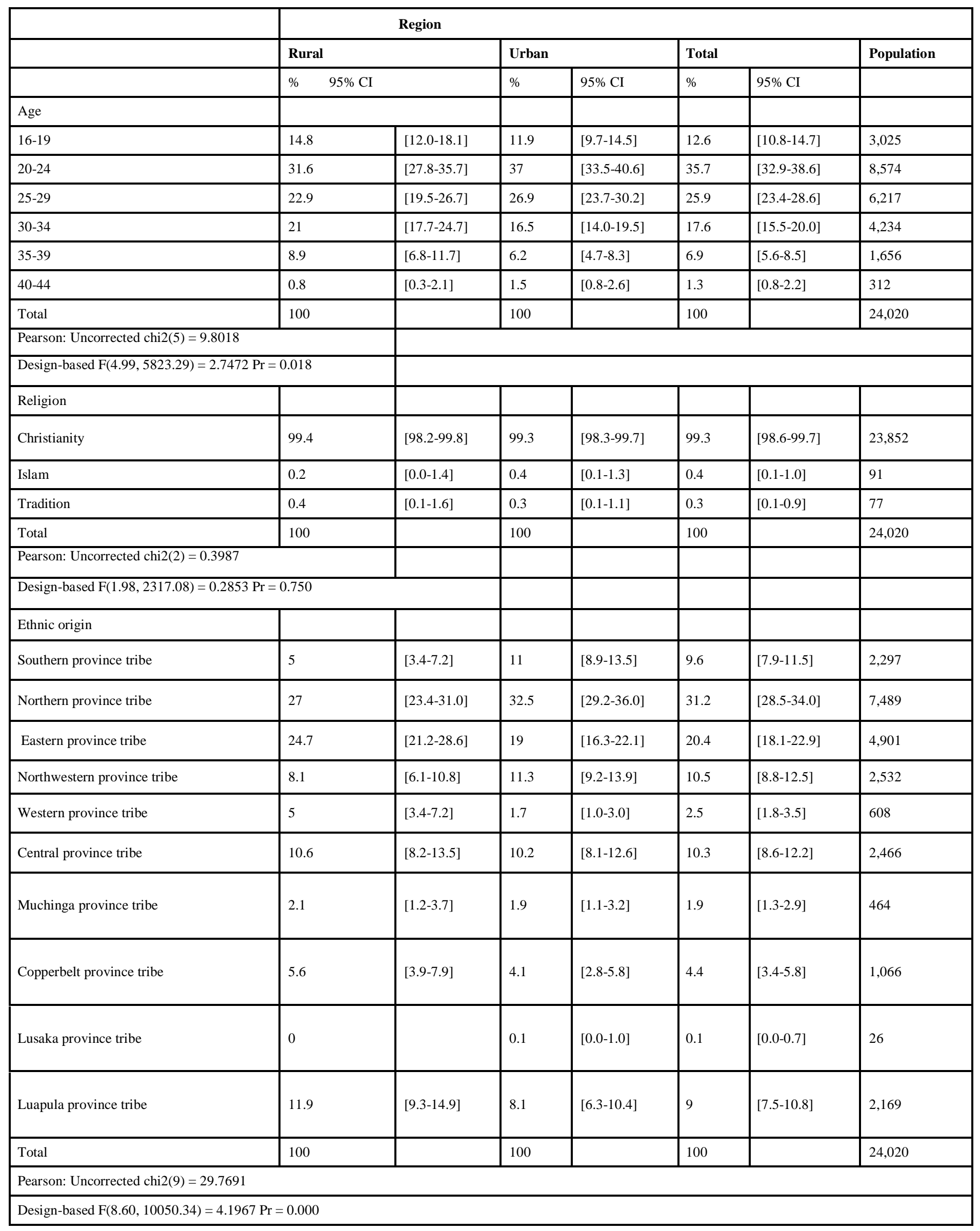




\begin{tabular}{|c|c|c|c|c|c|c|c|}
\hline Marital status & & & & & & & \\
\hline Married & 92.1 & {$[89.5-94.1]$} & 90.9 & {$[88.5-92.8]$} & 91.2 & {$[89.3-92.7]$} & 21,896 \\
\hline Casual/cohabiting & 1.5 & {$[0.7-2.9]$} & 1 & {$[0.5-2.1]$} & 1.1 & {$[0.7-1.9]$} & 270 \\
\hline Single & 5.8 & {$[4.1-8.2]$} & 7.8 & {$[6.1-10.1]$} & 7.3 & {$[5.9-9.1]$} & 1,765 \\
\hline Divorced or Separated & 0.6 & {$[0.2-1.8]$} & 0.3 & {$[0.1-1.1]$} & 0.4 & {$[0.1-0.9]$} & 89 \\
\hline Total & 100 & & 100 & & 100 & & 24,020 \\
\hline \multicolumn{8}{|l|}{ Pearson: Uncorrected chi2(3) $=2.2403$} \\
\hline \multicolumn{8}{|c|}{ Design-based $\mathrm{F}(2.98,3481.88)=0.9884 \mathrm{Pr}=0.397$} \\
\hline \multicolumn{8}{|l|}{ Education } \\
\hline Never attended school & 6.4 & {$[4.6-8.9]$} & 3.5 & {$[2.4-5.1]$} & 4.2 & {$[3.2-5.5]$} & 1,009 \\
\hline Primary level & 33.7 & {$[29.8-37.8]$} & 28.9 & {$[25.7-32.3]$} & 30 & {$[27.4-32.8]$} & 7,217 \\
\hline Secondary level & 53.4 & [49.1-57.7] & 55 & {$[51.3-58.6]$} & 54.6 & {$[51.7-57.6]$} & 13,121 \\
\hline Vocational/Technical/Professional & 6.4 & {$[4.6-8.9]$} & 12.6 & {$[10.4-15.3]$} & 11.1 & {$[9.4-13.2]$} & 2,673 \\
\hline Total & 100 & & 100 & & 100 & & 24,020 \\
\hline \multicolumn{8}{|l|}{ Pearson: Uncorrected chi2(3) $=13.6222$} \\
\hline \multicolumn{8}{|c|}{ Design-based $\mathrm{F}(2.98,3478.69)=6.2857 \mathrm{Pr}=0.000$} \\
\hline \multicolumn{8}{|l|}{ Occupation } \\
\hline $\begin{array}{l}\text { Teacher, Health worker, Office } \\
\text { Administrative worker }\end{array}$ & 3.5 & [2.3-5.5] & 3.3 & [2.2-4.9] & 3.4 & {$[2.5-4.6]$} & 813 \\
\hline Housewife/unemployed & 65.5 & {$[61.3-69.4]$} & 71 & {$[67.5-74.2]$} & 69.6 & {$[66.9-72.3]$} & 16,728 \\
\hline Trader & 18.3 & {$[15.2-21.8]$} & 12.2 & {$[10.0-14.8]$} & 13.7 & {$[11.8-15.8]$} & 3,284 \\
\hline Street vendor & 4.4 & {$[2.9-6.5]$} & 5.4 & [3.9-7.3] & 5.1 & {$[4.0-6.6]$} & 1,231 \\
\hline Fishmonger & 0.2 & {$[0.0-1.4]$} & 0.1 & {$[0.0-1.0]$} & 0.2 & {$[0.0-0.7]$} & 39 \\
\hline Farmer & 2.5 & {$[1.5-4.2]$} & 2.2 & [1.3-3.5] & 2.3 & {$[1.5-3.3]$} & 541 \\
\hline Hairdresser & 0.8 & {$[0.3-2.1]$} & 1 & {$[0.5-2.1]$} & 1 & {$[0.5-1.8]$} & 233 \\
\hline Seamstress & 0.2 & {$[0.0-1.4]$} & 0.6 & {$[0.2-1.5]$} & 0.5 & {$[0.2-1.2]$} & 118 \\
\hline Caterer & 0.2 & {$[0.0-1.4]$} & $\overline{0}$ & & 0.1 & {$[0.0-0.3]$} & 12 \\
\hline Other (specify) & 4.4 & [2.9-6.5] & 4.2 & {$[3.0-6.0]$} & 4.2 & {$[3.2-5.6]$} & 1,020 \\
\hline Total & 100 & & 100 & & 100 & & 24,020 \\
\hline \multicolumn{8}{|l|}{ Pearson: Uncorrected chi2(9) $=9.9245$} \\
\hline Design-based $F(8.92,10414.39)=1.596$ & $5 \operatorname{Pr}=$ & & & & & & \\
\hline
\end{tabular}

Table 1: showing socio-economic and demographic characteristics of pregnant women according region in the study population

\section{Environmental and behavioral characteristics relating to exposure}

Almost all $(94.5 \%)$ the pregnant women were involved in cooking duties during pregnancy and majority $(82.5 \%)$ cooked on a daily basis compared to only $7.9 \%$ who cooked occasionally. Most of the pregnant women spent one to four hours on cooking. Out of those involved in cooking during pregnancy, less than a third $(28.7 \%)$ spent the whole cooking period in the cooking area and close to three quarters $(43.0 \%)$ stayed in the cooking area half of the cooking period. More than half $(52.3 \%)$ of the participants reported using charcoal and less than a quarter $(14.8 \%)$ reported using wood. With regard to kitchen type, more than one third $(40.1 \%)$ pregnant women cooked in an enclosed kitchen, more than a quarter (16.9\%) in a semienclosed shelter and slightly more than one fifth $(20.3 \%)$ in an open space. Chimney was not common, only $3.5 \%$ reported presence of a chimney. Out of all those who cooked in an enclosed environment, $11.6 \%$ had no window while $74.0 \%$ and $12.1 \%$ reported having one and two windows respectively and out of those with windows, $74.3 \%$, $18.1 \%$ and $7.3 \%$, classified their windows as small, medium and very small respectively.
Close to two thirds of the participants $(61.6 \%)$ agreed that garbage burning was done at household level and $23.6 \%$ reported always present when garbage burning is taking place, others $24.1 \%, 26.5 \%$ and $25.8 \%$ reported mostly present, occasionally present and never present at all respectively. Of those who burn garbage at home $39.8 \%$ reported occasional burning while $19.1 \%$ of them reported burning 2-3 days/week and $14.8 \%$ burn daily. Majority $(79.6 \%)$ of the pregnant women reported that no one smoked at home and only $15.2 \%$ reported having one person smoking at home. Most of them (80.4\%) reported not being exposed to second hand smoke at home. More than half of pregnant women $(52.7 \%)$ reported spending 1- 4 hours outdoor and 28.9\% reported spending 5-8 hours outdoor per day. More than a third (38.4\%) women agreed to conducting their outdoor activities near an air pollution source and more than two quarters $(62.4 \%)$ described the air pollution source as a busy road (traffic hot spot) while $19.3 \%$ of them described it as a combustion site and for the $14.5 \%$ it was a waste disposal site. Table 2 shows the summary of the exposure characteristics for both rural and urban areas. 


\begin{tabular}{|c|c|c|c|c|c|c|c|}
\hline \multicolumn{8}{|l|}{ Region } \\
\hline & \multicolumn{2}{|c|}{ Rural } & \multicolumn{2}{|c|}{ Urban } & \multicolumn{2}{|c|}{ Total } & \multirow[t]{2}{*}{ Population } \\
\hline & $\%$ & $95 \% \mathrm{CI}$ & $\%$ & $95 \% \mathrm{CI}$ & $\%$ & $95 \% \mathrm{CI}$ & \\
\hline \multicolumn{8}{|c|}{ Cooking in pregnancy } \\
\hline Yes & 95 & [92.8-96.6] & 94.3 & [92.4-95.8] & 94.5 & [93.0-95.7] & 22,699 \\
\hline No & 5 & {$[3.4-7.2]$} & 5.7 & {$[4.2-7.6]$} & 5.5 & {$[4.3-7.0]$} & 1,321 \\
\hline Total & 100 & & 100 & & 100 & & 24,020 \\
\hline \multicolumn{8}{|c|}{ Pearson: Uncorrected chi2(1) $=0.1863$} \\
\hline \multicolumn{8}{|c|}{ Design-based $F(1.00,1168.00)=0.2672 \operatorname{Pr}=0.605$} \\
\hline \multicolumn{8}{|c|}{ Frequency of cooking } \\
\hline Daily & 81 & {$[77.4-84.2]$} & 82.9 & [79.9-85.5] & 82.5 & {$[80.1-84.6]$} & 19,157 \\
\hline 4-5 days/week & 2.4 & {$[1.3-4.1]$} & 2.8 & {$[1.8-4.4]$} & 2.7 & {$[1.9-3.9]$} & 635 \\
\hline 2-3 days/week & 4.5 & {$[3.0-6.7]$} & 5.4 & {$[3.9-7.4]$} & 5.2 & {$[4.0-6.7]$} & 1,205 \\
\hline Once/week & 3 & [1.8-4.9] & 1.3 & {$[0.7-2.5]$} & 1.8 & [1.1-2.7] & 407 \\
\hline Occasional & 9.1 & {$[6.8-11.9]$} & 7.5 & {$[5.8-9.7]$} & 7.9 & [6.4-9.6] & 1,829 \\
\hline Total & 100 & & 100 & & 100 & & 23,233 \\
\hline \multicolumn{8}{|c|}{ Pearson: Uncorrected chi2(4) $=4.4966$} \\
\hline \multicolumn{8}{|c|}{ Design-based $\mathrm{F}(3.98,4489.00)=1.5278 \operatorname{Pr}=0.192$} \\
\hline \multicolumn{8}{|c|}{ Time spent on cooking } \\
\hline 1 Hour & 16.1 & [13.2-19.6] & 17.4 & {$[14.7-20.4]$} & 17.1 & {$[14.9-19.5]$} & 3,971 \\
\hline 2 Hours & 24.5 & {$[21.0-28.5]$} & 23.4 & [20.4-26.7] & 23.7 & {$[21.2-26.3]$} & 5,500 \\
\hline 3 Hours & 20.2 & {$[16.9-23.9]$} & 16.5 & {$[13.9-19.4]$} & 17.4 & {$[15.2-19.8]$} & 4,043 \\
\hline 4 Hours & 23 & {$[19.5-26.9]$} & 29.2 & [26.0-32.7] & 27.7 & {$[25.1-30.5]$} & 6,445 \\
\hline 5 Hours & 3.9 & {$[2.5-6.0]$} & 0.7 & {$[0.3-1.8]$} & 1.5 & {$[1.0-2.3]$} & 350 \\
\hline 6 Hours & 11.6 & {$[9.1-14.7]$} & 10.9 & {$[8.8-13.5]$} & 11.1 & [9.4-13.1] & 2,582 \\
\hline 8 Hours & 0.6 & {$[0.2-1.9]$} & 1.8 & {$[1.0-3.1]$} & 1.5 & {$[0.9-2.5]$} & 353 \\
\hline Total & 100 & & 100 & & 100 & & 23,245 \\
\hline \multicolumn{8}{|c|}{ Pearson: Uncorrected chi2(6) $=20.2225$} \\
\hline \multicolumn{8}{|c|}{ Design-based $F(5.87,6632.37)=4.5579 \operatorname{Pr}=0.000$} \\
\hline \multicolumn{8}{|c|}{ Time spent in cooking area } \\
\hline Whole duration & 28.5 & {$[24.7-32.5]$} & 28.7 & {$[25.5-32.2]$} & 28.7 & {$[26.0-31.5]$} & 6,693 \\
\hline Half period & 41.2 & {$[37.0-45.5]$} & 43.6 & {$[39.9-47.3]$} & 43 & {$[40.0-46.0]$} & 10,034 \\
\hline Quarter period & 10.2 & {$[7.9-13.1]$} & 7.5 & {$[5.7-9.7]$} & 8.1 & {$[6.7-9.9]$} & 1,902 \\
\hline \multirow[t]{3}{*}{ Less than quarter } & 17.6 & {$[14.6-21.2]$} & 18.3 & {$[15.6-21.3]$} & 18.1 & {$[15.9-20.5]$} & 4,227 \\
\hline & 1.3 & {$[0.6-2.7]$} & 0.9 & {$[0.4-2.0]$} & 1 & {$[0.5-1.8]$} & 231 \\
\hline & 1.3 & {$[0.6-2.7]$} & 1 & {$[0.5-2.2]$} & 1.1 & {$[0.6-1.9]$} & 258 \\
\hline Total & 100 & & 100 & & 100 & & 23,344 \\
\hline \multicolumn{8}{|c|}{ Pearson: Uncorrected chi2 $(5)=2.6190$} \\
\hline \multicolumn{8}{|c|}{ Design-based $F(5.00,5680.07)=0.7124 \operatorname{Pr}=0.614$} \\
\hline Fuel type & & & & & & & \\
\hline
\end{tabular}

Table 2: showing exposure characteristics of pregnant women in the study population 


\section{Lung function test results of the pregnant women in the study population}

Table 3 presents the proportions of spirometry results of pregnant women in the study population. Almost all the pregnant women (99.3\%) had an FEV1/FVC higher than $70 \%$ and close to two thirds $(60.5 \%)$ of pregnant women had a normal FEV1 while more than one third (38.4\%) recorded mild obstruction. Normal FVC was obtained in 35.6\% of pregnant women while mild reduction and moderate reduction was recorded in $37.4 \%$ and $26.9 \%$ of pregnant women respectively.

\begin{tabular}{|c|c|c|c|c|c|c|c|}
\hline & \multicolumn{4}{|c|}{ Region } & \multirow{2}{*}{\multicolumn{2}{|c|}{ Total }} & \\
\hline & \multicolumn{2}{|l|}{ Rural } & \multicolumn{2}{|c|}{ Urban } & & & \multirow[t]{2}{*}{ Population } \\
\hline & $\%$ & $95 \% \mathrm{CI}$ & $\%$ & $95 \% \mathrm{CI}$ & $\%$ & $95 \% \mathrm{CI}$ & \\
\hline \multicolumn{8}{|l|}{ FEV1/FVC } \\
\hline$<70$ & 0.4 & {$[0.1-1.6]$} & 0.7 & {$[0.3-1.7]$} & 0.7 & {$[0.3-1.4]$} & 156 \\
\hline 70 & 0.2 & {$[0.0-1.4]$} & 0 & & 0.1 & {$[0.0-0.3]$} & 12 \\
\hline$>70$ & 99.4 & {$[98.2-99.8]$} & 99.3 & [98.3-99.7] & 99.3 & {$[98.6-99.7]$} & 23,852 \\
\hline Total & 100 & & 100 & & 100 & & 24,020 \\
\hline \multicolumn{8}{|c|}{ Pearson: Uncorrected chi2(2) $=2.1597$} \\
\hline \multicolumn{8}{|c|}{ Design-based $F(1.98,2316.80)=1.8092 \operatorname{Pr}=0.164$} \\
\hline \multicolumn{8}{|l|}{ FEV1 } \\
\hline Moderate Obstruction & 0 & & 1.5 & {$[0.8-2.6]$} & 1.1 & {$[0.6-2.0]$} & 264 \\
\hline Mild Obstruction & 34.1 & {$[30.2-38.3]$} & 39.8 & {$[36.2-43.4]$} & 38.4 & {$[35.5-41.3]$} & 9,221 \\
\hline Normal & 65.9 & {$[61.7-69.8]$} & 58.8 & {$[55.1-62.3]$} & 60.5 & {$[57.6-63.4]$} & 14,535 \\
\hline Total & 100 & & 100 & & 100 & & 24,020 \\
\hline \multicolumn{8}{|c|}{ Pearson: Uncorrected chi2(2) $=7.7284$} \\
\hline \multicolumn{8}{|c|}{ Design-based $\mathrm{F}(1.88,2194.03)=4.0867 \mathrm{Pr}=0.019$} \\
\hline \multicolumn{8}{|l|}{$\overline{\mathrm{FVC}}$} \\
\hline Moderate Reduction & 28.1 & {$[24.4-32.1]$} & 26.6 & {$[23.4-29.9]$} & 26.9 & {$[24.4-29.6]$} & 6,468 \\
\hline Mild Reduction & 34.7 & {$[30.8-38.9]$} & 38.3 & [34.8-41.9] & 37.4 & {$[34.6-40.4]$} & 8,994 \\
\hline Normal & 37.2 & {$[33.2-41.4]$} & 35.1 & {$[31.7-38.7]$} & 35.6 & [32.8-38.5] & 8,559 \\
\hline Total & 100 & & 100 & & 100 & & 24,020 \\
\hline \multicolumn{8}{|c|}{ Pearson: Uncorrected chi2 $(2)=1.1883$} \\
\hline Design-based F(2.00, 2 & $=0.83$ & .435 & & & & & \\
\hline
\end{tabular}

Table 3: showing proportions of spirometry results of pregnant women in the study population.

\section{Mean differences of lung functions between rural and urban area}

The mean FEV1/FVC were 96.69\% (SD 0.64) and 104.2\% (SD 0.43) respectively observed in rural and urban area. The two means varied significantly with a p-value of $<0.0001$. The mean FVC between rural and urban did not vary significantly while the mean FEV1 in the rural $(84.75 \%$ SD 0.44$)$ and urban $(83.07 \%$ SD 0.39$)$ varied significantly with the p-value 0.003 . Table 5 below presents the mean differences of the lung functions between rural and urban area.

\begin{tabular}{|c|c|c|c|c|c|c|c|}
\hline Factor & Group & Obs & Mean & Std. Dev. & [95\% Conf. & Interval] & P-value \\
\hline \multirow[t]{4}{*}{ FEV1/FVC } & Rural & 481 & 96.69 & 14.08 & 95.42 & 97.95 & \\
\hline & Urban & 687 & 104.2 & 11.22 & 103.4 & 105.1 & \\
\hline & Combined & 1168 & 101.1 & 13.01 & 100.4 & 101.9 & $<0.0001$ \\
\hline & $\overline{\text { Diff }}$ & & -7.53 & & -8.98 & -6.07 & \\
\hline \multirow[t]{4}{*}{ FVC } & Rural & 481 & 75.89 & 7.857 & 75.18 & 76.59 & \\
\hline & Urban & 689 & 75.74 & 7.767 & 75.16 & 76.32 & \\
\hline & Combined & 1170 & 75.8 & 7.801 & 75.35 & 76.25 & 0.374 \\
\hline & Diff & & 0.149 & & -0.76 & 1.059 & \\
\hline \multirow[t]{4}{*}{ FEV1 } & Rural & 481 & 84.75 & 9.706 & 83.88 & 85.62 & \\
\hline & Urban & 689 & 83.07 & 10.32 & 82.3 & 83.84 & \\
\hline & combined & 1170 & 83.76 & 10.1 & 83.18 & 84.34 & 0.003 \\
\hline & diff & & 1.684 & & 0.51 & 2.859 & \\
\hline
\end{tabular}

Table 5: showing mean differences of spirometry together with the standard deviations, and p-values at $95 \%$ confidence interval between rural and urban area. 
Factors associated with a declined lung function at bivariate analyses in rural and urban areas

Table 6 below present's results of the bivariate analysis and only one factor showed a statistically significant association with declined lung function. Cooking fuel was statistically significantly associated with a declined lung function ( $\mathrm{p}$ - value 0.005 ) while gravida only showed a weak association with declined lung function at $\mathrm{p}$ value 0.056 .

\begin{tabular}{|c|c|c|c|c|c|c|c|}
\hline & \multicolumn{2}{|l|}{ Rural } & \multicolumn{2}{|c|}{ Urban } & \multicolumn{2}{|l|}{ Total } & \multirow[b]{2}{*}{$P$ value } \\
\hline Factor & (n) & $\%$ & (n) & $\%$ & (n) & $\%$ & \\
\hline \multicolumn{7}{|l|}{ Age } & \multirow[t]{7}{*}{0.157} \\
\hline 16-19 & (154) & 13.07 & (119) & 13.11 & (35) & 13.07 & \\
\hline $20-24$ & (406) & 34.78 & (326) & 36.17 & (80) & 30.01 & \\
\hline $25-29$ & (296) & 25.18 & (225) & 24.88 & (71) & 26.18 & \\
\hline $30-34$ & (215) & 18.36 & $(165)$ & 18.37 & (50) & 18.37 & \\
\hline 35-39 & $(87)$ & 7.39 & (59) & 6.35 & (27) & 10.49 & \\
\hline $40-44$ & (14) & 1.18 & (9) & 1.01 & (5) & 1.85 & \\
\hline \multicolumn{7}{|l|}{ Education } & \multirow[t]{5}{*}{0.752} \\
\hline Not attended & (56) & 4.67 & (44) & 4.85 & (11) & 4.07 & \\
\hline Primary & (361) & 30.85 & (271) & 30.14 & (89) & 33.27 & \\
\hline Secondary & $\begin{array}{l}(493 \\
)\end{array}$ & 54.69 & (143) & 53.17 & (636) & 54.38 & \\
\hline College & (94) & 10.32 & (25) & 9.38 & (118) & 10.07 & \\
\hline $\begin{array}{l}\text { Cookingfuel } \\
\text { choice }\end{array}$ & & & & & & & \multirow[t]{6}{*}{0.005} \\
\hline Charcoal & $(622)$ & 53.2 & $(475)$ & 52.58 & (147) & 55.09 & \\
\hline Wood & (229) & 19.6 & (176) & 19.47 & (53) & 19.06 & \\
\hline $\begin{array}{l}\text { Crop } \\
\text { residues }\end{array}$ & (29) & 2.5 & (17) & 1.87 & (12) & 4.45 & \\
\hline Electricity & (118) & 10.16 & (106) & 11.58 & (14) & 5.18 & \\
\hline Combination & (172) & 14.6 & (128) & 14.39 & (41) & 15.36 & \\
\hline $\begin{array}{l}\text { Cooking } \\
\text { environment }\end{array}$ & & & & & & & \multirow[t]{4}{*}{0.540} \\
\hline Enclosed & $(504)$ & 43.22 & (385) & 42.57 & (121) & 44.87 & \\
\hline $\begin{array}{l}\text { Semi- } \\
\text { detached }\end{array}$ & $(508)$ & 43.28 & (399) & 44.21 & (107) & 40.37 & \\
\hline Open space & (158) & 13.51 & (119) & 13.24 & (39) & 14.58 & \\
\hline \multicolumn{7}{|l|}{ Gravida } & \multirow[t]{3}{*}{0.056} \\
\hline $\begin{array}{l}\text { Prime } \\
\text { gravida }\end{array}$ & (271) & 23.24 & (223) & 24.62 & (50) & 18.68 & \\
\hline Multigravida & (899) & 76.81 & $(680)$ & 75.43 & (217) & 81.32 & \\
\hline $\begin{array}{ll}\text { Body } & \text { Mass } \\
\text { Index } & \\
\end{array}$ & & & & & & & \multirow[t]{6}{*}{0.881} \\
\hline Underweight & $(517)$ & 44.10 & $(402)$ & 44.52 & (116) & 42.70 & \\
\hline Overweight & (164) & 14.10 & (125) & 13.81 & (40) & 15.02 & \\
\hline Obese & (72) & 6.21 & (56) & 6.24 & (15) & 6.11 & \\
\hline Severe obese & (49) & 4.20 & (40) & 4.43 & (9) & $\mathbf{3 . 4 2}$ & \\
\hline Normal & (368) & 31.51 & $(\mathbf{2 8 0})$ & 31.12 & (87) & $\mathbf{3 . 3 1}$ & \\
\hline Blood Pressure & & & & & & & 0.909 \\
\hline High BP & (36) & 3.13 & $(26)$ & 3.12 & (9) & $\begin{array}{l}3.3 .0 \\
1\end{array}$ & \\
\hline Normal BP & $\begin{array}{l}(113 \\
4)\end{array}$ & 96.91 & $\overline{(877)}$ & 97.01 & $(\mathbf{2 5 8})$ & 96.7 & \\
\hline
\end{tabular}

Table 6: Bivariate Logistic Regression: Showing factors associated with declined lung function .
Association between cooking fuel choices and declined lung function at Multivariate analyses in rural and urban

After running a multivariate analysis to determine the association between declined lung function and the type of cooking fuel, the findings indicate there was a statistical significant relationship between cooking fuel of choice and declined maternal lung function. Compared with pregnant women who use combined type (charcoal and electricity), pregnant women using crop residues as cooking fuel are two times more likely to have a declined lung function [AOR $2.33(1.27,4.30)]$ and those who use electricity only are $57 \%$ less likely to have declined lung function [AOD $0.43(0.26,0.69)]$. Table 7 summarizes the findings of the multivariate logistic regression.

\begin{tabular}{|l|l|}
\hline Factor & $\begin{array}{l}\text { Adjusted Odds Ratios (95\% } \\
\text { C.I) }\end{array}$ \\
\hline & \\
\hline Cooking fuel choices & \\
\hline Combined (charcoal and electricity) & 1 \\
\hline Charcoal & $1.00(0.78,1.29)$ \\
\hline Wood & $0.99(0.72,1.36)$ \\
\hline Crop residue & $2.33(1.27,4.30)$ \\
\hline Electricity & $0.43(0.26,0.69)$ \\
\hline & \\
\hline $\begin{array}{l}\text { Constant: } 0.28 \\
\text { Variable(s) entered on step1: fuel, gravida }\end{array}$ \\
\hline
\end{tabular}

Table 7: Multivariate Logistic Regression: cooking fuels associated with a declined lung function

\section{Discussion}

Our study findings indicate a high biomass use for cooking among pregnant women in the study population. Compared to the urban areas, rural areas recorded the highest level of biomass use, an observation that is comparable with most sub-Saharan African countries where previous studies indicate consistent results of high level of solid fuel use for cooking especially in the rural areas when compared with the urban areas $[22,23]$. The use of solid fuel as a primary source of household energy is consistent with the findings in previous studies in other developing countries, a study in Ethiopia observed that solid fuel in form of crop residues, firewood and animal dung was the primary source of household energy [24] in most households.

In our study, use of mixed fuel for cooking showed no adverse effect on respiratory functions compared to the use of biomass only. However, a contrasting result from a study conducted in India found that women using mixed fuel experienced more respiratory symptoms, followed by biomass, stove and LPG users [25]. Our current study found that use of crop residue for cooking was more likely to cause a declined lung function compared to use of mixed type of cooking fuel. This finding is supported by the fact that the efficiency of cooking with cleaner fuels is higher compared with crop residues. Crop residue is at the bottom of the energy ladder and this is one of the reasons that commercial fuels such as electricity or LPG are considered to be superior to crop residue and dung [26]. The fact that crop residues are more inefficient than other fuel types, it also follows that they emit high levels of pollutants compared to other fuel types, increasing the risk of poor lung function among the women using this type of cooking fuel. Women who used electricity only for cooking in our study were less likely to have poor lung function result compared to those who used mixed fuel types. This is an expected finding, however, it is sad to note that only $12.4 \%$ of the study population reported use of electricity only for cooking. The large majority use solid fuels such wood, charcoal, crop residue and mixed (charcoal and electricity). This scenario is comparable to many other countries in sub-Saharan Africa [20]. 
A study conducted in Addis Ababa reports that traditional fuels (wood, charcoal and dung) meet about $75 \%$ of household energy needs in Ethiopia and the remaining $25 \%$ is provided by kerosene, LPG and electricity [27,28]. Unlike Ethiopia where, the price of electricity has declined by more than $50 \%$, being sold at approximately $75 \%$ less than the price of kerosene [29], the price of electricity in Zambia is on the higher side for an average Zambian household and most households that have electricity in their houses prefer to use it for lighting and not cooking as cooking is said to consume a lot of energy leading to unaffordable high electricity bills [30] (Zambia Energy Sector Report, 2014).

A strong correlation between use of biomass fuel for cooking and reduced pulmonary function was recorded in our study. This finding is in accord with a large body of evidence [11,20,30,31,32]. However, it is also important to note that reduction in pulmonary function has not been consistently documented by all researchers. In a previous study to assess the impact of different cooking fuels on pulmonary function among nonsmoking women, Reddy el al observed no correlation between biomass cooking fuel and pulmonary function [22]. The researchers attributed the absence of the expected adverse effects of biomass on pulmonary functions to better ventilation in the cooking environments of women in the biomass group compared to previous studies that support our findings.

\section{Conclusion}

The findings of our study proved evidence that use of cooking fuel options that are at the bottom of the energy ladder is a strong determinant of declined lung function. The availability and affordability of cleaner fuels in both rural and urban areas should be improved through promoting and harnessing technologies that are important in the development of affordable, high efficient and lowemission cooking fuel options.

\section{References}

1. International Energy Agency (2002) World Energy Outlook, Chapter 13, Energy and Poverty ITDG Reducing Indoor Air Pollution in Rural Households in Kenya: Working With Communities to Find Solutions (ITDG Project 1998-2001)

2. WHO (2006) Fuel For Life: Household Energy and Health, Geneva

3. Ezzati M, Kammen, DM (2001) Indoor air pollution from biomass combustion and acute respiratory infections in Kenya: an exposure-response study. Lancet 358, 619e629.

4. Chidumayo, EN (2002) Changes in Miombo Woodland Structure under Different Land Tenure and Use Systems in Central Zambia. Journal of Biogeography 29: 1619-1626.

5. Indaba Agricultural Policy Research Institute (IAPRI) (2015) Cooking Fuel Choice in Urban Zambia: Implications on Forest Cover. Lusaka, Zambia.

6. Smith KR, Aggarwal, AL, Dave RM (1983) Air pollution and rural biomass fuels in developing countries: a pilot village study in India and implications for research and policy. Atmos. Environ. 17 (11), 2343e2362.

7. ICMR (2001) Bulletin: Indoor air pollution in India-A major environmental and public health concern, ICMR Bulletin, 31.

8. Bihari V, Iqbal SM, Srivastava LP, Kesavachandran C, Siddique MJA (2013) Lung function impairment in women exposed to biomass fuels during cooking compared to cleaner fuels in Uttar Pradesh, India. Journal of Environmental Biology; vol.34,971-974)

9. Rehfuess E (2006) Fuel for Life: Household Energy and Health, World Health Organization, Geneva.

10. World Health Organization (2004) Global Burden of Disease Due to Indoor Air Pollution.
11. Rinne ST, Rodas EJ, Bender BS, Rinne ML, Simpson JM, GalerUnti R, et al. (2006) Relationship of pulmonary function among women and children to indoor air pollution from biomass use in rural Ecuador. Respir Med.100:1208-15.

12. Pope D, Diaz E, Smith-Sivertsen T, Lie RT, Bakke P, Balmes JR, Kirk R, Smith KR, and Bruce NG. Exposure to Household Air Pollution from Wood Combustion and Association with Respiratory Symptoms and Lung Function in Nonsmoking Women: Results from the RESPIRE Trial, Guatemala. Environ Health Perspect;2016;124(3):A48.

13. Kurmi OP, Semple S, Simkhada P, Smith WC, Ayres JG (2010) COPD and chronic bronchitis risk of indoor air pollution from solid fuel: a systematic review and meta-analysis. Thorax 65:221-228.

14. Naeher LP, Smith KR, Leaderer BP, Nuefeld L, Mage DT (2001) Carbon monoxide as a tracer for assessing exposures to particulate matter in wood and gas cookstove households of highland Guatemala. Environ Sci Technol.35:575-581.

15. WHO Air Quality Guidelines for Particulate Matter, Ozone, Nitrogen Dioxide and Sulphur Dioxide, Global Update (2005) (2006) Summary of RiskAssessment. Geneva: WHO.

16. Ozkaynak H, Xue J, Spengler JJ, Wallace L, Pellizzari E and Jenkins P (1995) "Personal exposure to airborne particles and metals: Results from the particle team study in riverside, California," J. Exposure Anal. Environ. Epidemiol., vol. 6, no. 1, pp. 57-78.

17. Clayton C, Perritt RL, Pellizzari ED, Thomas KW, Whitmore RW, Wallace LA et al. et al (1992) "Particle total exposure assessment methodology (PTEAM) study: Distributions of aerosol and elemental concentrations in personal, indoor, and outdoor air samples in a southern california community," J. Exposure Anal. Environ. Epidemiol., vol. 3, no. 2, pp. 227-250.

18. Sheldon L. et al (1989) "An investigation of infiltration and indoor air quality," New York State Energy Res. Develop. Authority, Albany, NY, USA, Tech. Rep. NYSERDA-90-11 ON: T191001298.

19. Sarnat J. A., Koutrakis P., and Suh H. H., "Assessing the relationship between personal particulate and gaseous exposures of senior citizens living in Baltimore,MD," J. Air Waste Manage. Assoc., vol. 50, no. 7, pp. 1184-1198.

20. Desalu OO, Adekoya AO (2010) Ampitan BA. Increased risk of respiratory symptoms and chronic bronchitis in women using biomass fuels in Nigeria. J Bras Pneumol.36:441-6.

21. Reddy TS, Guleria R., Sanjeev Sinha, Sharma SK and Pande JN (2004) Domestic Cooking Fuel and Lung Functions in Healthy Non-smoking Women. Indian J Chest Dis Allied Sci; 46 : 85-90

22. Ezzati M, Kammen D (2001) Indoor air pollution from biomass combustion and acute respiratory infections in Kenya: An exposureresponse study. Lancet; 358:619-24.

23. Fullerton DG, Semple S, Kalambo F, Suseno A, Malamba R, Henderson G, et al. (2009) Biomass fuel use and indoor air pollution in homes in Malawi. Occup Environ Med.66:777-83.

24. Kumie A, Emmelin A, Wahlberg S, Berhane Y, Ali A, Mekonen E (2009) Worku A, Brandstrom D. Sources of variation for indoor nitrogen dioxide in rural residences of Ethiopia. Environmental health (8):51

25. Behera D, Jindal SK (1991) Respiratory symptoms in Indian women using domestic cooking fuels. Chest.100:385-8.

26. IARC. Household Use of Solid fuels and High-temperature Frying (2010) http://monographs.iarc.fr/. Accessed 23.03.2018

27. Central Statistical Agency: The Federal Democratic Republic of Ethiopia. Welfare Monitoring Survey 2004. Analytical Report. (2004) Addis Ababa. Central Statistical Agency

28. Central Statistical Agency and ORC Macro: Ethiopia demographic and health survey 2005. 2006, Addis Ababa, Ethiopia and Calverton, Maryland, USA. Central Statistical Agency and ORC Macro. 
29. Sanbata H, Asfaw A, Kurmie A (2014) indoor air pollution in slum neighbourhoods of Addis Ababa, Ethiopia. Atmospheric Environment 89 230e234.

30. Zambia Energy Sector Report, 2014. available on: http://www.erb.org.zm/reports/EnergySectorReport2014.pdf Accessed on 23.03.2018
31. Regalado J, Pérez-Padilla R, Sansores R, Páramo Ramirez JI, Brauer M, Paré P, Vedal S (2006)The effect of biomass burning on respiratory symptoms and lung function in rural Mexican women.Am J Respir Crit Care Med. Oct 15;174(8):901-5. Epub 2006 Jun 23.

32. Smith-Sivertsen T, Diaz E, Pope D, Lie RT, Díaz A, McCracken J, et al.( 2009) Effect of reducing indoor air pollution on women's respiratory symptoms and lung function: the Respire Randomized Trial, Guatemala. J Am Epidemiol;170:211-20. 